\title{
Photostability Characterization of Wood Polymer Composites of Polyvinyl Chloride and Rice Husk to Ultra-Violet Irradiation Exposure
}

\author{
Farhana Hazwanee M.Jais ${ }^{1, ~}{ }^{*}$, Nurzhatul Aziemah A. Omar ${ }^{1}$, and Anika Zafiah M. Rus ${ }^{1}$ \\ ${ }^{1}$ Sustainable Polymer Engineering (E1), Advanced Manufacturing and Materials Center (AMMC), \\ Faculty of Mechanical and Manucfacturing Engineering,University Tun Hussein Onn Malaysia, \\ Malaysia
}

\begin{abstract}
In this study, the photostability of wood polymer composites (WPC) was assessed by using ultra-violet accelerated weathering test. Meanwhile, the mechanical properties of WPC show strength improvement at prolonged UV irradiation exposure with $43.21 \mathrm{MPa}$ and maximum strain of $4.08 \%$ at 5000 hours and 1000 hours UV irradiation exposure respectively. The colour stability of WPC was improved by addition of Ultraviolet (UV) stabilizer during pre-mixing process which shows positive effect on the colour stability and prevented chalking of the composites for external use. Generally, discolourations of WPC during test exposure were caused by degradation of both wood and plastic. Therefore, incorporation with pigments and other additives gives improvement to the photostability of WPC. This is based on the increment of WPCs mechanical property while the morphological fracture surface of dumbbell test specimens revealed the pull out rice husk fiber which contributed to the distribution of load in the WPC samples.
\end{abstract}

\section{Introduction}

Polymers are high molecular weight substances consisting of molecules that are approximately multiples of simple units [1]. The word polymer comes from the Greek poli, which means many, and meros, which means parts ${ }^{1}$. A polymer is called a plastic when it has other materials such as stabilizers, plasticizers, or other additives are in it [1].

WPCs are produced starting from rice husk (up to $50 \%$ ) in the form of fibres, mixed with a polymeric matrix polyvinyl chloride and additives such as coupling agents, plasticizer, UV stabilizer and lubricant to improve the physical and mechanical characteristic of polymer [2]. Interfacial adhesion between rice husk and thermoplastics is usually very weak due to the high interfacial tension which leads to undesirable properties of the composites. The process of modifying interfacial properties of an incompatible polymer blend leading to the production of polymer blend is called compatibilization [3].

* Corresponding author: farhana.irmb@gmail.com 
In WPCs, the composites of wood and plastic basically contain several materials are added in small amounts to affect the performance and processing. Coupling agents, calcium zinc stabilizer, plasticizer, polyethylene wax and also titanium dioxide as an additives are all used to some extent in improving physical properties.

Coupling agents are used to improve the bonding between the hydrophilic wood fiber and hydrophobic matrix. This has been the most active area of research in employing an additive to provide moisture resistance to WPCs. Coupling agents can reduce the amount of moisture WPCs absorb by reducing gaps at the wood matrix interface and by reducing the number of hydroxyl groups available for hydrogen bonding with water. However, the decreases in water absorption can be minor. Steckel et al. [4] examined the moisture sorption properties of several WPCs using a full factorial design and determined that wood flour content had the largest influence on equilibrium moisture content, followed by removal of the original polymer rich composite surface.

The inclusion of a coupling agent and reducing particle size had a much smaller effect on equilibrium moisture content. Panthapulakkal et al. [5] examined water absorption of 65 $\%$ rice husk filled HDPE composites. After immersion in water for 1608 hours the control composites absorbed $14 \%$ water. Composites containing a coupling agent absorbed either $12 \%$ or $9 \%$ depending on the coupling agent used. However, the use of a lubricant as a processing aid negated any positive effect of the coupling agent. Lin et al. [6] reported an $18 \%$ decrease in moisture absorption when $2 \%$ coupling agent was added to $15 \%$ wood flour filled PP composite. Coupling agents may be more effective at mitigating changes in strength and modulus after exposure to cyclical freeze-thaw testing. The increase in weight change of rice husk filled HDPE composites was $9.6 \%$ after 12 freeze-thaw cycles. Adding a coupling agent resulted in increases of 3.3-3.5\% of polymer strength [5].

UV irradiation exposure test can be used to study the polymer photostability, and can provide data regarding polymer life time. This test is important due to the photostability characteristic of polymer will influence the polymer commercial success. Furthermore, UV irradiation is one of the factors that can affect the polymer degradation process [7]. The two most popular UV sources for photo-degrading materials have been the xenon arc and fluorescent lamps. Both the xenon arc and fluorescent light are gas discharge tubes and are usually line source.

\section{Methodology}

The experiment of characterization and analysis were conducted on virgin polyvinyl chloride (VPVC), rice husk (RH), plasticizer, coupling agents, calcium zinc stabilizer, polyethylene wax, and titanium dioxide $\left(\mathrm{TiO}_{2}\right)$. Virgin PVC and $\mathrm{RH}$ were prepared based on addition of thermoplastic doped with Titanium Dioxide $\left(\mathrm{TiO}_{2}\right)$ and additives consist of coupling agents, plasticizer, lubricant and stabilizer known as dry blend. The dry blend was then mixed using high speed hot mixer at certain temperature and discharged to cooling mixer to bring down the dry blend's temperature. The dry blend was then fed into pelletizing feeder to produce WPCs pellet at certain parameter setting.

The WPCs pellets produced was then run on conical twin screw extruder to fabricate WPCs profile for further analysis. The WPCs sample fabricated was analyzed under scanning electron microscope and tensile strength samples preparation is using Injection moulding machine. The setting parameter of Injection moulding machine with nozzle temperature is $200^{\circ} \mathrm{C}$, middle is $180^{\circ} \mathrm{C}$, front is $195^{\circ} \mathrm{C}$, rear 1 is $175^{\circ} \mathrm{C}$, rear 2 is $165^{\circ} \mathrm{C}$ and feeds is $55^{\circ} \mathrm{C}$ as shown in Figure 1. 
The WPCs samples were exposed under UV light in the UV Weatherometer to examine its tensile properties which examine the photostability of WPC. Higher improvement of WPC strength property is indicating higher stability of the samples under harsh environmental condition after UV weatherometer. Meanwhile the fracture morphological surface of tensile test sample of WPCs was analyzed using scanning electron microscope (SEM).

\section{Tensile Test of Ultra Violet Irradiation of Wood Polymer Composites}

The mechanical property of WPC is conducted such as to measure its strength of polymers and its filler. Tensile test is used to determine the tensile strength, modulus of elasticity and elongation at break. The tensile test samples are prepared according to ISO 527-2 (5A) as Figure 1. The test is conducted by using Universal Tensile Machine AG-I, Shimadzu, with $10 \mathrm{kN}$ of force. The plotted results in Figure 2 revealed that the tensile strength were increased after uv irradiation exposure at 1000 hours but started to decrease at 5000 hours.

(a)

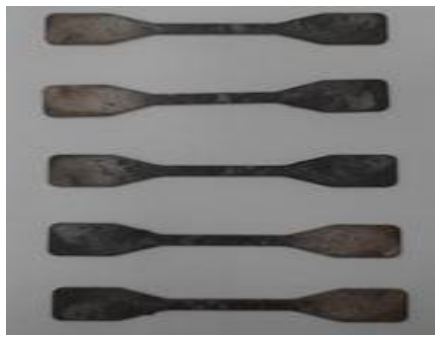

(b)

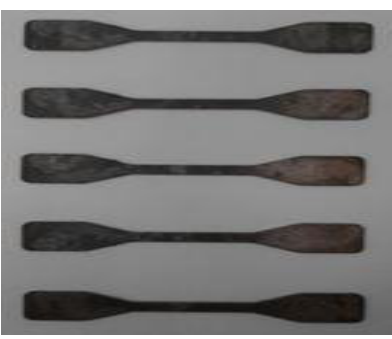

Fig. 1. Dumbell specimen of WPC (a) unirradiated WPC samples, (b) WPC of 5000 hours UV irradiated.

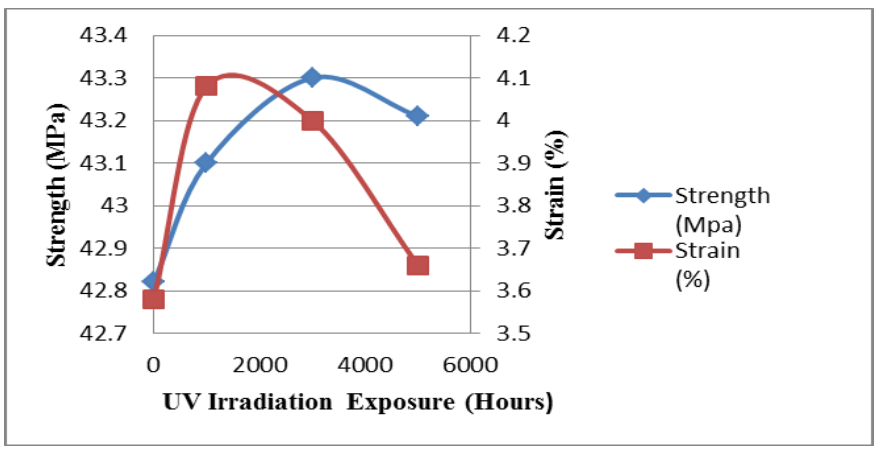

Fig. 2. Mechanical strength and strain of WPC samples of UV irradiation exposure (hours).

It can be seen that the nonlinear tensile behaviour to break is highly sensitive to structural changes in the polymer due to UV light exposure. The ultimate mechanical properties, and particularly strain at break of various polymer systems, react to the photooxidative degradation much earlier and in a more pronounced way than in the usual physicochemical analytic methods. Thus strain at break can be utilized in practice as a sensitive indicator of the degree of degradation of photo-oxidized polymers by chemicrystallization. 
The photo-oxidation causes molecular chain scissions in the amorphous regions and these parts of cut chains rearrange into a crystalline phase. Thus, the reduction of mechanical properties is related to the increase of the crystallinity fraction. Additionally, the strain value of polymer compound was increased with the increasing of UV irradiation exposure hours as shown in Figure 2. It is observed that the strain has increased with prolonged UV irradiation exposure with the maximum value of strain is $4.08 \%$. It is believed that the polymer content, cross-linker and UV irradiation have influenced the bond strength of the polymer. Thus, the variations in strength and strain may be due to the changes in chain-scission/cross-linking ratio that occurred during UV irradiation exposure of the polymer compounds.

\section{Scanning Electron Microscopy (SEM) of Wood Polymer Composites of Ultra-Violet Irradiation Exposure}

SEM is used to evaluate the changes in fracture surface morphology of polymer compound of WPC. The cross-section of fracture surfaces of samples are coated with platinum by using JFC-1600 auto fine coater and result as Table 1. The tensile specimens' fractures are observed by using scanning electron microscope of model Jeol JFM-6380 located at Material Characterization Laboratory, FKMP, UTHM. The SEM micrograph fracture surfaces of WPC shows the elongation break of filler appears after pulling out when stress is applied. The morphology of miscible polymer compound depends on the components, ratios and processing conditions. The PVC samples indicated that this polymer is contributed to high tensile strain.

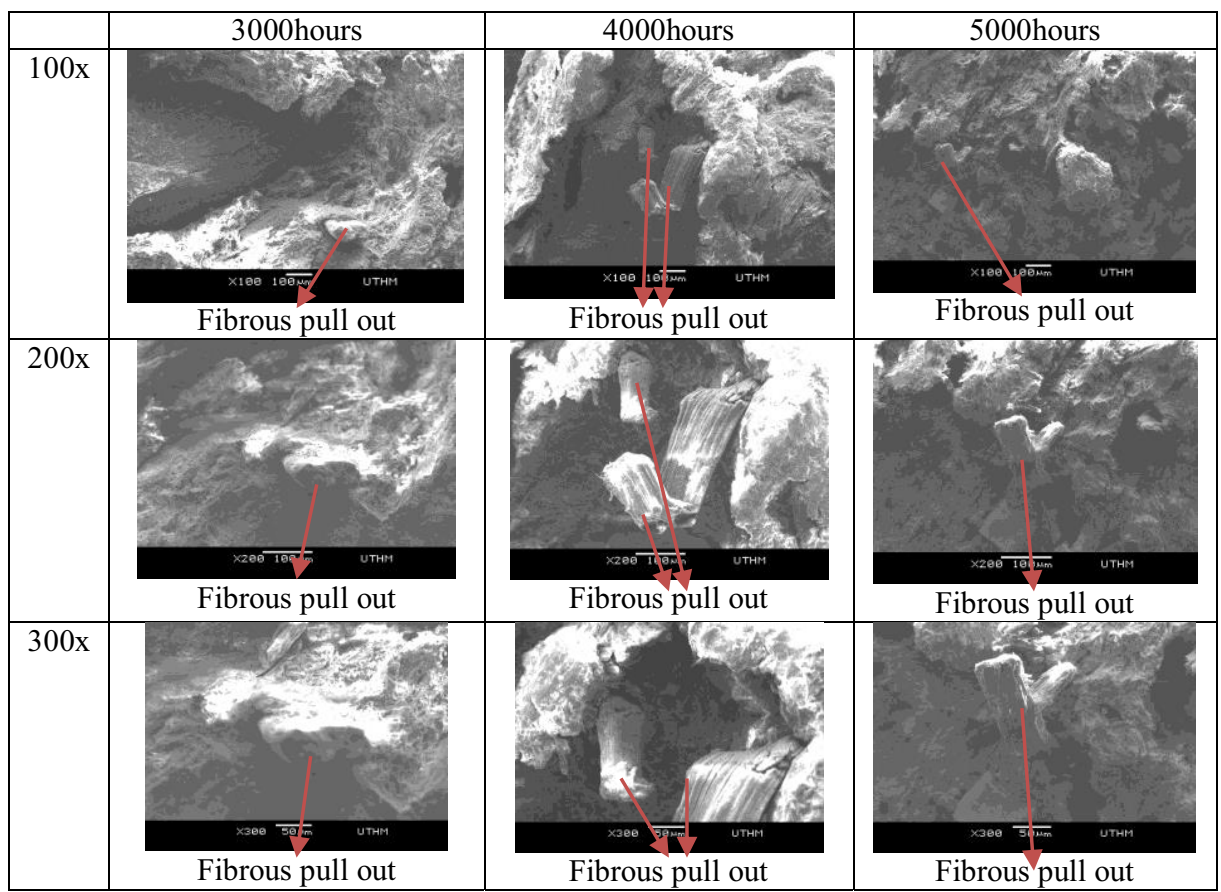

Fig. 3. Comparison between SEM cross section of fracture surface of WPC tensile specimens samples for 3000 hours, 4000 hours and 5000 hours. 
Figure 3 shows fibrous pull out from fracture surface of WPC tensile specimen which contributed to stress distribution through the tensile specimen of the wood fiber and polymer matrix. These specimens experienced brittle interfaced failure. Weak interlayer bonding or some amount of interlayer porosity was evident in the failure of the specimens and appeared to be the cause of layer delamination along to the WPC during loading [8]. This is also contributed from the miscible polymer compound which depends on the materials composition ratios and processing condition during the fabrication process.

\section{Conclusions}

Wood polymer composites of polyvinyl chloride (PVC) with rice husk have good mechanical and physical properties. The addition of the rice husk in PVC was affecting the physical and mechanical properties of WPC during UV irradiation exposure. The WPC shows increment in strength gradually at 5000 hours of UV irradiation exposure. This is due to the crosslinking of polymer and increased interphases connection between polymer, rice husk fibres and other additive.

The authors would like to thank the Malaysian Government and Universiti Tun Hussein Onn Malaysia (UTHM), Johor, for supporting this research study under Exploratory Researched Grant Scheme, ERGS Vot E018. Not to forget, the authors express deep gratitude to ZFH Industries Sdn Bhd for supplying the pellet used in this research study.

\section{References}

1. C. Clemons, Wood-Polymer Composites (Woodhead Publishing Limited, 2008)

2. D. Ndiaye, E. Fanton, S.M. Therias, L. Vidal, A.Tidjani, J.L. Gardette, Compos. Sci. Technol., 68, 2779 (2008)

3. H. Nasution, Green Building Materials and Civil Engineering (CRC Press, Taylor \& Francis Group, London, 2015)

4. V. Steckel, C.M. Clemons, H. Thoemen, J. Appl. Polym. Sci., 103, 752 (2007)

5. S. Panthapulakkal, S. Law, M. Sain, J. Thermoplast. Compos. Mater., 18, 445 (2015)

6. Q. Lin, X. Zhou, G. Dai, J. Appl. Polym. Sci., 85, 2824 (2002)

7. J. Tocháček, Z. Vrátníčková, Polym. Test., 36, 82 (2014)

8. C. Ziemian, M. Sharma, S. Ziemi, Mechanical Engineering (Science, Technology and Medicine, 2012) 